\title{
Comparison of the efficacy and safety of mometasone furoate dry powder inhaler to budesonide Turbuhaler $\mathbb{R}$
}

\author{
J. Bousquet*, A. D'Urzo**, J. Hebert***, C.H. Barraza ${ }^{+}$, L-P. Boulet ${ }^{\#}$, R. Suárez-Chacón ${ }^{++}$,

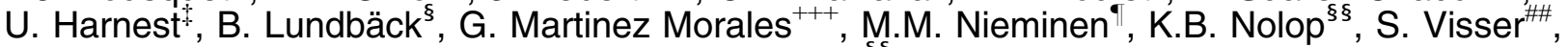 \\ B.N. Lutsky ${ }^{\S \S}$
}

Comparison of the Efficacy and Safety of Mometasone Furoate Dry Powder Inhaler to BudesonideTurbuhaler $(\mathbb{B}$. J. Bousquet, A. D'Urzo, J. Hebert, C.H. Barraza, L-P. Boulet, R. Suárez-Chacón, U. Harnest, B. Lundbäck, G. Martinez Morales, M.M. Nieminen, K.B. Nolop, S. Visser, B.N. Lutsky. ERS Journals Ltd. 2000.

ABSTRACT: Mometasone furoate (MF) administered by dry powder inhaler (DPI) was composed with budesonide (BUD) Turbuhaler $(\mathbb{B}$ in the treatment of moderate persistent asthma.

The patients were randomized to one of four treatment groups: MF DPI $(100,200$, $400 \mu \mathrm{g}$ b.i.d) or BUD Turbuhaler $\mathrm{R} .400 \mu \mathrm{g}$ b.i.d in a 12-week, active-controlled, evaluator-blind, multicentre international trial. The primary efficacy variable was the mean change from baseline to endpoint (last treatment visit) in forced expiratory volume in one second (FEV1).

Changes in FEV1 showed a statistically significant superiority $(p<0.05)$ of MF DPI 200 and $400 \mu \mathrm{g}$ b.i.d compared with the BUD Turbuhaler $\AA 400 \mu \mathrm{g}$ b.i.d treatment. Significant superiority $(\mathbf{p}<\mathbf{0 . 0 5})$ was also seen in scores for several secondary efficacy variables when MF DPI was compared with BUD Turbuhaler $\mathbb{R}$ treatment. MF DPI $200 \mu \mathrm{g}$ b.i.d was comparable to MF DPI $400 \mu \mathrm{g}$ b.i.d in therapeutic benefit. The incidence of oral candidiasis was no more than $3 \%$ in any group. All treatments were well tolerated.

A total daily dose of $\mathbf{4 0 0} \mu \mathrm{g}$ of mometasone furoate administered by dry powder inhaler provides a well-tolerated treatment for patients with moderate persistent asthma and results in a significantly greater improvement, when compared to a daily dose of $800 \mu \mathrm{g}$ BUD Turbuhaler $B$ in the parameters measured in this study. Eur Respir J 2000; 16: 808-816.

\begin{abstract}
*Hôpital Arnaud de Villenueve, Montpellier Cedex, France. **Primary Care Asthma Clinic, Toronto, Canada. ***Place de la Cite, Quebec, Canada. ${ }^{+}$Hospital Clinica del Parque, Chihuahua, Mexico. "Hôpital Laval, Quebec, Canada. ${ }^{++}$Policlinica Metropolitana, Caracas, Venezuela. tNymphenburger Strasse, München, Germany. ${ }^{\S}$ Medical Dept, Bodens sjukhus, Boden, Sweden. ${ }^{++}$Hospital General de Enfermedad Comun, I.G.S.S., Guatemala, CA, USA. "Tampere University Hospital, Finland. ${ }^{\text {s }}$ SSchering-Plough Research Institute, Kenilworth, NJ, USA. "\# Dept Internal Medicine, H. F. Verwoerd Hospital, Pretoria, South Africa.
\end{abstract}

Correspondence: J. Bousquet Hôpital Arnaud de Villeneuve Service des Maladies Respiratories 555, route de Ganges 34059 Montpellier Cedex France Fax: 011 33467042708

Keywords: Asthma, budesonide, corticosteroid, glucocorticoid, Mometasone Furoate, Turbuhaler $®$

Received: June 201999

Accepted after revision July 152000

Supported by the Schering-Plough Research Institute.
Inhaled corticosteroids are very effective in controlling asthma and are recommended for the long-term control of mild, moderate and severe persistent asthma according to national and international guidelines [1-3]. Identification of new potent inhaled corticosteroids with minimal systemic bioavailability would provide added safety for the treatment of patients with persistent asthma.

Mometasone furoate (MF) is a potent topical glucocorticoid in both preclinical and clinical studies. In vitro studies demonstrated it to be highly effective in the inhibition of cytokine production [4-6], as well as being one of the most potent stimulators tested for glucocorticoid receptor-mediated gene transcription, with a very high affinity for the glucocorticoid receptor [7]. MF is effective and well tolerated as a nasal spray in the control of seasonal allergic and perennial rhinitis [8-10]. More recently, a dry powder inhaler (DPI) formulation of MF has been developed for the treatment of asthma. Anhydrous MF formulated as micronized agglomerates with inhalation grade lactose (5.8 parts lactose: 1 part MF) is delivered via a compact breath-actuated DPI device, utilizing cap activation technology for dose metering. MF DPI was designed to promote compliance via its ease of use (a three-step operation) and to deliver an accurate dose over a range of inspiratory flow rates for adults and children over $12 \mathrm{yrs}$ with mild to severe persistent asthma. Studies show a dose-to-dose variability of less than $10 \%$ at inspiratory flow rates of $20-60 \mathrm{~L} \cdot \mathrm{min}^{-1}[11]$. The MF DPI was shown to be very effective in the treatment of mild to moderate persistent asthma $[12,13]$.

While the overall risk for developing adverse systemic effects is considerably less with inhaled corticosteroids compared with oral steroid therapy, exposure to high doses of some currently available agents can cause detectable systemic effects [14], such as suppression of the hypothalamic-pituitary-adrenal axis. The clinical significance of the detected systemic effects of inhaled corticosteroids is controversial; it may be relevant, however, for some patients [15-17].

The clinical efficacy of MF has not previously been directly compared with budesonide (BUD), an inhaled corticosteroid which is commonly used to treat patients 
with moderate persistent asthma. This randomized, evaluator-blind, active-controlled study compared the efficacy and safety of MF DPI in doses of 100, 200, and 400 $\mu \mathrm{g} b . i . d$ to a single dose of BUD $(400 \mu \mathrm{g}$ by Turbuhaler $\AA$, b.i.d) in patients who were previously maintained on daily inhaled corticosteroids for treatment of their moderate persistent asthma. These doses of MF were chosen based on the results from a previous study [12] that indicated efficacy and safety of MF across this dose range in a population of adult and adolescent patients with moderate persistent asthma. The $400 \mu \mathrm{g}$ b.i.d dose of BUD

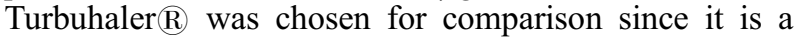
dose commonly used worldwide to treat patients with moderate persistent asthma.

\section{Methods}

\section{Patients}

Adults or adolescents ( $\geq 12$ yrs of age) of either sex, who had a history of asthma for at least six months and who had been using an inhaled glucocorticoid daily for at least 30 days, were eligible to participate in the study. Prior to screening and through to baseline, patients must have been maintained on a stable regimen of inhaled corticosteroid, including flunisolide, triamcinolone acetonide, beclomethasone dipropionate, BUD or fluticasone propionate. Patients had to demonstrate a baseline forced expiratory volume in one second (FEV1) $60 \%-90 \%$ of predicted normal value after all restricted medications had been withheld for specified intervals. In addition, they had to demonstrate reversibility of airway disease by an increase in FEV1 of $\geq 12.0 \%$ over the pre-bronchodilator value, with an absolute volume increase of at least 200 $\mathrm{mL}$, within $30 \mathrm{~min}$ after two inhalations of salbutamol (Ventolin (B)). All patients were nonsmokers or had stopped smoking more than six months prior to screening. Clinical laboratory values, 12-lead electrocardiograms (ECGs) and vital signs were all to be clinically acceptable. All patients were free of any clinically significant disease other than asthma.

Patients were excluded for any of the following reasons: premenarche, pregnancy, lactation, requiring allergenspecific immunotherapy, unless on a stable maintenance schedule, treatment with oral corticosteroids for $>14$ days in the six months prior to screening, treatment with methotrexate, cyclosporine or gold within three months, or systemic steroids or another investigational drug in the month prior to screening, daily use of more than $1 \mathrm{mg}$ of nebulized $\beta_{2}$-adrenergic agonists (either metered dose inhaler (MDI) or inhaled powder, depending on the preference of the study site), use of any long-acting $\beta_{2^{-}}$ adrenergic agonist less than 2 weeks prior to screening, the need for ventilator support in the past five yrs, hospitalization for asthma in the last three months, use of $>12$ puffs day $^{-1}$ of salbutamol on any two consecutive days between screening and baseline visits, treatment for asthma in an emergency room or admission to a hospital for management of airway obstruction, on two or more occasions in the past six months, clinical evidence of significant pulmonary disease other than asthma, a history of glaucoma and/or posterior subcapsular cataracts, increase or decrease in FEV1 of $\geq 20 \%$ between screening and baseline visits, any clinically relevant abnormal baseline vital sign, any clinically significant abnormal ECG or chest radiograph at screening or within the previous month, any respiratory tract infection during the two weeks prior to screening, evidence of clinically significant oropharyngeal candidiasis. An acceptable method of birth control was required for all women of childbearing potential.

\section{Study design}

This randomized, evaluator-blind, active-controlled study compared the efficacy and safety of MF DPI (100, 200, and $400 \mu \mathrm{g}$ b.i.d) and BUD powder administered as Pulmicort $\AA$ Turbuhaler $(4)(400 \mu \mathrm{g} b . i . d)$. The study was double-blind with respect to MF treatment groups, as the identities of the MF DPI devices were unknown to patients and investigators. Patients randomized to MF were instructed to take one inhalation from each DPI (i.e. either one active and one placebo, or two active DPIs) in the morning and again in the evening. The two dosage strengths were indistinguishable from each other and from the placebo DPI device. Similarly, patients randomized to BUD were instructed to take one inhalation from each of the two Turbuhaler $(\mathbb{R})$ devices morning and evening.

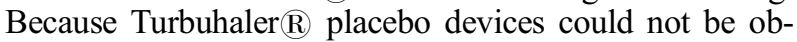
tained for double-dummy blinding, the study was evaluator-blind as to whether a patient received MF DPI or BUD Turbuhalerß). In all cases, either a separate pharmacy or drug distribution centre or an individual not otherwise associated with the conduct of the study was identified for the patient and was used to distribute study medication to the patient. Patients were specifically instructed not to discuss any aspect of their study medication with anyone else associated with the study; all questions regarding use or malfunction of study medications were restricted to those individuals dispensing medications, because of the evaluator-blind nature of the study.

The study was conducted at 57 study centres in 17 countries. Randomization was generated in a $1: 1: 1: 1$ : ratio with a block size of 4 . A random code was generated for each country, and patients were assigned sequentially as they entered each study centre within the country. The study did not contain a placebo treatment group since placebo treatment of patients with moderate persistent asthma is considered unethical in most of the countries in which this study was conducted. An Ethics Committee reviewed the protocol for each centre and each patient, and a parent or guardian for patients $<18$ yrs of age also provided written informed consent.

Medications prohibited after the screening visit included those linked with clinically significant hepatotoxicity or induction of liver enzymes, beta blockers, nasal, ocular, oral and inhaled corticosteroids (except for inhaled glucocorticoid therapy during the run-in period), dermatologic corticoids other than mild category, oral decongestants, oral or inhaled bronchodilators (other than salbutamol), nedocromil or cromolyn sodium, astemizole and leukotriene modifiers. The last doses of oral betaadrenergic bronchodilators were withheld for $24 \mathrm{~h}$ (syrups and tablets) or $48 \mathrm{~h}$ (sustained-release tablets), and longacting beta-adrenergic bronchodilators (e.g. salmeterol) were withheld for 2 weeks prior to pulmonary function tests at screening and prohibited thereafter. Short-acting inhaled or nebulized $\beta_{2}$-adrenergic agonists were withheld six $\mathrm{h}$ before any study visit. Overuse of rescue medication 
could result in discontinuation from the study. Theophylline was permitted throughout the study if a stable dose was an established part of the patient's therapeutic regime prior to the screening visit.

During the run-in period, patients received treatment with their normally prescribed inhaled corticosteroid. At the baseline visit, patients eligible for participation in the study discontinued use of their previous inhaled corticosteroid and were randomly assigned to one of the following treatments: MF DPI 100, 200, or $400 \mu \mathrm{g}$ b.i.d; or BUD Turbuhaler $\mathrm{R} 400 \mu \mathrm{g}$ b.i.d. All treatments were taken twice a day, in the morning and again in the evening, approximately $12 \mathrm{~h}$ apart. Patients were instructed to rinse their mouths with water or mouthwash following drug administration.

All patients completed daily diary cards to document morning (a.m.) and evening (p.m.) peak expiratory flow rate (PEFR), recording the highest of triplicate efforts for each. They also recorded salbutamol use, asthma symptoms, number of night-time awakenings requiring salbutamol use, adverse events, and use of study drug and concomitant medications. Daily scores or values were averaged over weekly intervals. Treatment visits occurred after 1,2, 4, 8, and 12 weeks of treatment. At all treatment visits, pulmonary function, including FEV1 and forced vital capacity (FVC), was measured by spirometry, and investigators performed an oropharyngeal exam for the presence of candidiasis, reviewed diary cards, and assessed response to therapy. Treatment compliance was evaluated at each visit by someone other than the blinded evaluator by direct inquiry of the patient and review of the diary data. Use of $<75 \%$ or $>125 \%$ of the protocol-specified doses constituted a protocol deviation. Ten patients ( 5 in the MF DPI $100 \mu \mathrm{g}$ b.i.d, 2 in the $200 \mu \mathrm{g}$ b.i.d, 2 in the $400 \mu \mathrm{g}$ b.i.d and 1 in the BUD $400 \mathrm{mg}$ b.i.d groups) were excluded from the efficacy evaluation subset for poor compliance (all took $<75 \%$ of specified doses). Compliance in the use of rescue medication was also evaluated at each visit by someone other than the blinded evaluator. This included an objective assessment of doses used, as well as a review of the patient' $\mathrm{s}$ diary data reports.

\section{Efficacy assessments}

The primary efficacy variable was the change from baseline to endpoint (the last evaluated treatment visit) in FEVl. Secondary efficacy variables included changes from baseline in the FVC, PEFR, symptom scores, nocturnal awakenings requiring salbutamol use as rescue medication, daily salbutamol use, and physician evaluation of response to therapy. Asthma symptom severity was rated on a 4point scale $(0=$ none; $1=$ noticeable; $2=$ annoying, some interference with daily activities; $3=$ very uncomfortable, interference with daily activities). Physicians rated response to therapy comparing current symptom level to level at baseline on a 5 -point scale $(1=$ much improved; $2=$ improved; $3=$ no change; $4=$ worse; $5=$ much worse).

\section{Safety assessments}

All patients were monitored for adverse events as well as for changes in vital signs and haematologic, urinalysis and blood chemistry profiles during the 12 -week treatment period. Plasma samples obtained at $08: 00 \mathrm{~h} \pm 1 \mathrm{~h}$ were analyzed for cortisol levels by radioimmunoassay at screening and week 12. Clinically significant asthma worsening required discontinuation from the study. This included $\mathrm{a} \geq 20 \%$ decrease in FEV1 from the baseline value or an asthma exacerbation that resulted in emergency treatment, hospitalization, or treatment with asthma medication in addition to those allowed on study. A clinically significant increase in bronchodilator use was defined as more than $1.6 \mathrm{mg}$ use on 2 consecutive days, or the lower total daily dose restricted by local labelling. A decrease of $\geq 25 \%$ in a.m. or p.m. PEF from baseline a.m. value on two consecutive days, or a clinically significant increase in bronchodilator use, were also considered to be an indicator of significant asthma worsening and led to discontinuation from the study. Investigators graded adverse events with regard to severity and investigators and patients assessed the relationship of any adverse event to the use of a study drug.

\section{Statistical analysis}

The study was designed to enroll at least 600 patients, or 150 patients per treatment group. This sample size was chosen to allow detection of a clinically meaningful difference in FEV1 of approximately $6 \%$ of the baseline value between any two treatment groups, with $80 \%$ power and $5 \%$ significance level, assuming a pooled standard deviation(SD) of 0.45 units for FEV1 change from baseline, based on earlier studies. The analyses of efficacy and safety were based on all the randomized patients who received at least one dose of study medication and who had post-baseline data (Intent-to-Treat principle). Endpoint was defined as the last observation obtained for an individual during the treatment period, to account for possible discontinuation of patients during the course of the study.

Predicted FEV1 was calculated, as appropriate, according to Crapo formulas [18] (for patients $\geq 18$ yrs of age), European Community Steel and Coal formulas (Nordic countries only, patients $18-70$ yrs of age) or the Polgar formula [19] (patients 12-17 yrs of age). Adjustments for patients of African descent were made by programming the spirometer or by hand calculation.

Changes from baseline FEV1, as well as the secondary efficacy variables, were analyzed using a two-way analysis of variance (ANOVA) that extracted sources of variation due to treatment and centre and treatment-by-centre interaction. Since all pairwise comparisons were of independent interest, each ANOVA was followed by Duncan's multiple range test to compare all treatment groups. The results of these tests are considered significant at the 0.05 level. The assessment of response to therapy as percentage of patients showing improvement or much improvement from baseline was analyzed by Fisher's Exact Test.

\section{Results}

\section{Patient population}

A total of 730 patients from 57 study centres in 17 countries were randomized after screening into treatment groups in this study. The description of the groups of all patients who received at least one dose of study medication is described in tables 1 and 2 (Intent-to-Treat groups). 
Table 1. - Demographics of the study population

\begin{tabular}{|c|c|c|c|c|}
\hline & \multicolumn{3}{|c|}{$\begin{array}{l}\text { MF DPI } \\
\mu \mathrm{g} \text { b.i.d }\end{array}$} & \multirow{2}{*}{$\begin{array}{c}\text { BUD } \\
\text { Turbuhaler } \mathbb{R} \\
\mu \mathrm{g} \text { b.i.d } \\
400\end{array}$} \\
\hline & 100 & 200 & 400 & \\
\hline Subjects $n$ & 185 & 176 & 188 & 181 \\
\hline \multicolumn{5}{|l|}{ Age yrs } \\
\hline Mean & 39 & 42 & 41 & 42 \\
\hline Range & $14-71$ & $14-76$ & $12-74$ & $12-76$ \\
\hline \multicolumn{5}{|l|}{ Sex } \\
\hline Male & 80 & 81 & 76 & 78 \\
\hline Female & 105 & 95 & 112 & 103 \\
\hline \multicolumn{5}{|l|}{ Ethnic group } \\
\hline White & 143 & 132 & 141 & 139 \\
\hline African & 1 & 2 & 4 & 2 \\
\hline Hispanic & 41 & 39 & 43 & 37 \\
\hline Asian & 0 & 2 & 0 & 3 \\
\hline Other & 0 & 1 & 0 & 0 \\
\hline \multicolumn{5}{|c|}{ Body weight $\mathrm{kg}$} \\
\hline Mean & 70 & 71 & 72 & 73 \\
\hline Range & $36-144$ & $41-135$ & $35-118$ & $34-131$ \\
\hline \multicolumn{5}{|c|}{$\begin{array}{l}\text { Smoking history } \\
\mathrm{n}(\%)\end{array}$} \\
\hline Never & $123(66)$ & $129(73)$ & $131(70)$ & $127(70)$ \\
\hline $\begin{array}{l}\text { Not in past } \\
6 \text { months }\end{array}$ & $61(33)$ & $46(26)$ & $57(30)$ & $52(29)$ \\
\hline
\end{tabular}

Treatment groups were comparable with respect to demographics (table 1) and asthma-related characteristics (table 2). These measures were assessed using descriptive statistics or the two-way ANOVA previously described, as appropriate. Statistics on treatment safety and efficacy were derived from this group on the "Intent-to-Treat" principle.
Patients enrolled in this study were maintained on prescribed inhaled corticosteroids prior to the initiation of the study. Both the number of patients using a particular prescribed glucocorticoid at baseline and the average dosage of that drug were similar among active treatment groups (table 2). The inhaled corticosteroids used included beclomethasone dipropionate (overall mean $\left.699 \mu \mathrm{g} \cdot \mathrm{day}^{-1}\right)$, budesonide $\left(662 \mu \mathrm{g} \cdot \mathrm{day}^{-1}\right)$, flunisolide $\left(659 \mu \mathrm{g} \cdot \mathrm{day}^{-1}\right)$, fluticasone propionate $\left(438 \mu \mathrm{g} \cdot \mathrm{day}^{-1}\right)$, or triamcinolone acetonide $\left(416 \mu \mathrm{g} \cdot \mathrm{day}^{-1}\right)$. The number of patients using theophylline at baseline and throughout the study was low and similar among treatment groups.

Overall, $14 \%$ of patients were $(n=101 / 730)$ discontinued from the study prior to completion and they were similarly distributed across treatment groups; $15 \%, 10 \%$, and $18 \%$ for the MF DPI 100, 200 and $400 \mu \mathrm{g}$ b.i.d groups, respectively and $14 \%$ for the budesonide Turbuhaler $\AA$ group. Treatment failure accounted for $5 \%$ overall $(n=$ 33/730). Discontinuations resulting from treatment failure were similar in the MF DPI $(5 \%, 3 \%$, and $6 \%$ for the 100 , 200 and $400 \mu \mathrm{g}$ b.i.d groups, respectively) or budesonide Turbuhaler $\AA$ (3\%) groups.

Adverse events were responsible for discontinuations in $2 \%$ of patients overall and these are described later (Evaluation of safety section). Other reasons for discontinuance were: noncompliance with protocol $(1 \%)$, lost to follow-up $(2 \%)$, did not meet entry criteria $(2 \%)$, or other reasons unrelated to treatment $(2 \%)$.

The requirement of both a baseline measurement and at least one post-baseline measurement was met by 720 patients, and efficacy analyses were based on this set. Some patients entered the study with FEV1 values or variability outside of the selection criteria (table 2 ). The number of such patients ranged from $2-7 \%$ for each

Table 2. - Asthma-related baseline characteristics of the study population

\begin{tabular}{|c|c|c|c|c|}
\hline & \multicolumn{3}{|c|}{ MF DPI $\mu \mathrm{g}$ b.i.d } & \multirow{2}{*}{$\begin{array}{c}\text { BUD Turbuhaler } \mathbb{R} \\
\mu \mathrm{g} \text { b.i.d } \\
400\end{array}$} \\
\hline & 100 & 200 & 400 & \\
\hline Subjects $n$ & 185 & 176 & 188 & 181 \\
\hline Duration of asthma yrs & $16(1-57)$ & $17(1-64)$ & $15(1-46)$ & $15(1-59)$ \\
\hline \multicolumn{5}{|l|}{ Respiratory function } \\
\hline FEV1 $\%$ Pred \pm SEM & $76.2 \pm 0.7$ & $77.1 \pm 0.8$ & $77.9 \pm 0.7$ & $76.0 \pm 0.7$ \\
\hline Range & $50-98$ & $55-113$ & $57-116$ & $55-110$ \\
\hline FEV1 L & 2.49 & 2.52 & 2.54 & 2.47 \\
\hline FVC L & 3.43 & 3.47 & 3.45 & 3.43 \\
\hline PEFa.m L. $\min ^{-1}$ & 398 & 385 & 390 & 381 \\
\hline \multicolumn{5}{|l|}{ Patient self-reports } \\
\hline Wheeze a.m.* & 0.31 & 0.47 & 0.43 & 0.35 \\
\hline Difficulty breathing a.m.* & 0.46 & 0.59 & 0.53 & 0.50 \\
\hline Cough a.m.* & 0.35 & 0.45 & 0.41 & 0.30 \\
\hline Nocturnal awakenings $\mathrm{n} *$ & 0.36 & 0.33 & 0.41 & 0.30 \\
\hline Salbutamol use $\mu \mathrm{g} \cdot$ day $^{-1}$ & 256 & 282 & 259 & 252 \\
\hline \multicolumn{5}{|l|}{ Inhaled glucocorticoid use $\mu \mathrm{g} / \mathrm{n}$} \\
\hline Beclomethsone dipropionate & $679 / 91$ & $695 / 86$ & $687 / 98$ & 736/98 \\
\hline Budesonide & $645 / 66$ & $688 / 66$ & $652 / 65$ & $663 / 654$ \\
\hline Flunisolide & $760 / 5$ & $750 / 4$ & $500 / 1$ & $625 / 4$ \\
\hline Fluticasone propionate & $430 / 22$ & $446 / 14$ & $452 / 22$ & $422 / 25$ \\
\hline Triamcinolone acetonide & $200 / 1$ & $550 / 4$ & $500 / 2$ & NA \\
\hline \multicolumn{5}{|l|}{ Theophylline use } \\
\hline Yes/No n & $17 / 168$ & $20 / 156$ & $17 / 171$ & $23 / 158$ \\
\hline
\end{tabular}

*: data are presented as mean score. FEV1: forced expiratory volume in one second; \% pred: per cent predicted; FVC: forced vital capacity; PEFa.m: peak expiratory flow in the morning. Values are presented as means and mean (range) where appropriate. 
group ( 3 patients in the MF $100 \mu \mathrm{g}$ b.i.d, 13 in the MF $200 \mu \mathrm{g}$ b.i.d, 13 in the MF $400 \mu \mathrm{g}$ b.i.d and 12 in the 400 $\mu \mathrm{g}$ BUD groups), for an overall total of $5.7 \%$. Only 2 patients failed the reversibility test (both in the MF 100 $\mu \mathrm{g}$ b.i.d group). The other principal reasons for protocol deviations were poor compliance $(<75 \%$ of specified doses taken, $n=10$ patients) and overuse of rescue medication ( $\mathrm{n}=8$ patients). Thus the majority of all protocol deviations were FEV1 deviations. Exclusion of FEV1 and other protocol deviations from the "Intent-to-Treat" set resulted in an efficacy evaluated subset of 654 patients.

\section{Evaluation of efficacy: forced expiratory volume in one second}

Baseline values were similar across groups for FEV1 (table 2). For analysis of treatment-by-centre interaction, centres with $<10$ patients were combined, within the same country when possible. The treatment-by-centre interaction term was not significant $(\mathrm{p}=0.14)$, while there was a significant treatment effect $(\mathrm{p}=0.03)$. For all treatment groups, substitution of study drug for the previously used inhaled corticosteroid was associated with improvement in lung function, as shown by the changes in FEV1 values over time (fig. 1). Both the MF DPI 200 and $400 \mu \mathrm{g}$ b.i.d groups showed significantly greater improvement com-

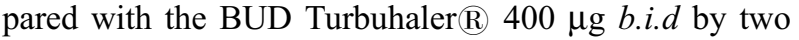
weeks and both doses generally maintained this difference throughout the study (fig. 1). Overall, increases in FEV1 values over time with BUD $400 \mu \mathrm{g}$ b.i.d were of smaller magnitude and, at week 8 , were actually significantly less than all three MF DPI b.i.d treatments.

The change in FEV1 from baseline to endpoint is indicated in table 3 . This change was significantly greater for MF DPI 200 and $400 \mu \mathrm{g}$ b.i.d than for BUD $400 \mu \mathrm{g}$ b.i.d by $0.10 \mathrm{~L}$. Likewise, the change in FEV1 from baseline for MF DPI $400 \mu \mathrm{g}$ b.i.d was significantly greater than that for MFD DPI $100 \mu \mathrm{g}$ b.i.d by $0.06 \mathrm{~L}$.

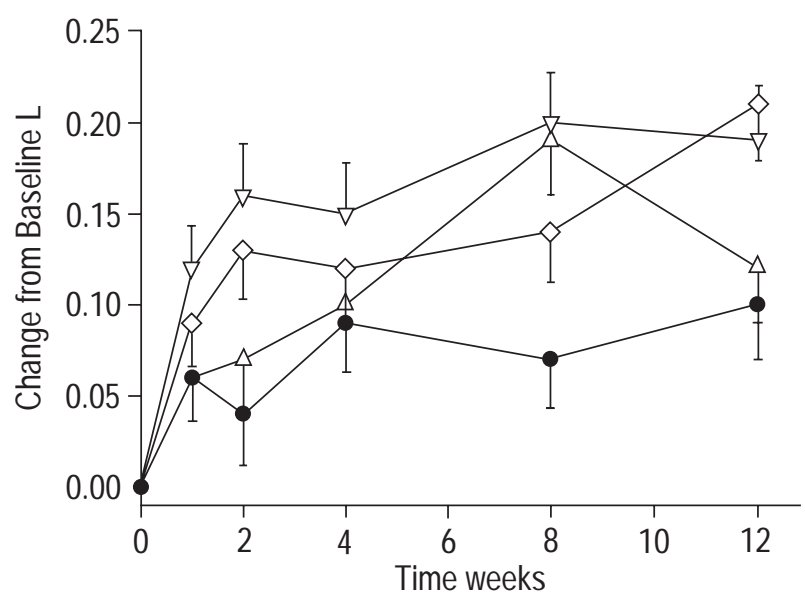

Fig. 1. - The effects of mometosone furoate administered by dry powder inhaler (MF DPI) b.i.d and budesonide (BUD) Turbuhaler® b.i.d on the mean change in forced expiratory volume in one second. The changes for MF DPI $100 \mu \mathrm{g}, 200 \mu \mathrm{g}, 400 \mathrm{mg}$ b.i.d and BUD Turbuhaler® $\mu \mathrm{g}$ b.i.d are indicated at weeks $1,2,4,8$ and 12. $(\triangle)$ : MF $100 \mu \mathrm{g}$ b.i.d; $(\nabla)$ : MF $200 \mu \mathrm{g}$ b.i.d; $(\diamond)$ : MF $400 \mu \mathrm{g}$ b.i.d; $(\diamond)$ : BUD $400 \mu \mathrm{g}$ b.i.d. Values are represented as mean \pm SEM.
Table 3 shows that FEV1, reported as percent of predicted (\% pred), increased from baseline to endpoint for all treatment groups. In addition, endpoint FEV1\% pred values of the groups treated with MF DPI $200 \mu \mathrm{g}$ b.i.d and MF DPI $400 \mu \mathrm{g}$ b.i.d were significantly greater than that of BUD $400 \mu \mathrm{g}$ b.i.d. Treatment with MF DPI $400 \mu \mathrm{g}$ b.i.d also produced a significantly higher endpoint FEV $1 \%$ pred value than treatment with MF DPI $100 \mu \mathrm{g}$ b.i.d. These data confirmed the inferences derived from comparison of changes from baseline in absolute FEV1 values.

Evaluation of efficacy: other pulmonary function measures

Additional measures of pulmonary function are summarized in table 2 , and responses to the study drugs are summarized in table 3.

Participants in the study measured a.m. and p.m. PEF each day prior to any study medication and recorded the number of puffs of salbutamol taken daily. Baseline values for a.m. PEF were similar among the treatment groups (table 2). The a.m. PEF was improved over time for all treatment groups (fig. 2 and table 3 ). The mean increase in a.m. PEF at the endpoint was significantly greater for patients treated with MF DPI $200 \mu \mathrm{g}$ b.i.d than with MF DPI $100 \mu \mathrm{g}$ b.i.d by $19.64 \mathrm{~L} \cdot \mathrm{min}^{-1}$. The increase for patients treated with MF DPI $400 \mu \mathrm{g}$ b.i.d was also significantly greater than with MF DPI $100 \mu \mathrm{g}$ b.i.d by 19.11 L. $\min ^{-1}$ (table 3). For p.m. PEF (data not shown) the mean increase at the endpoint was improved for MF DPI $400 \mu \mathrm{g}$ b.i.d when compared with MF DPI $100 \mu \mathrm{g}$ b.i.d treatment. There was no significant difference between the MF DPI 200 and $400 \mu \mathrm{g}$ b.i.d groups.

\section{Evaluation of asthma symptoms}

Patients rated asthma symptoms (wheezing, difficulty breathing and cough) each morning (a.m.) and evening (p.m.); scores were generally low at baseline in all treatment groups (table 2). Improvement in a.m. wheezing scores at the endpoint was significantly greater for patients in the MF DPI $400 \mu \mathrm{g}$ b.i.d treatment group than for those in either BUD Turbuhaler $\AA 400 \mu \mathrm{g}$ b.i.d or MF DPI $100 \mu \mathrm{g}$ b.i.d groups. Improvement in a.m. difficulty breathing at the endpoint was significantly greater for the MF DPI $400 \mu \mathrm{g}$ b.i.d group compared with the MF DPI $100 \mu \mathrm{g}$ b.i.d group. No significant differences were observed between treatment groups for a.m. coughing scores at the endpoint. The results for the p.m. asthma symptoms (data not shown) were generally similar to the a.m. results.

Daily use of salbutamol and nocturnal awakenings requiring use of salbutamol

Patients recorded use of salbutamol on their daily diary cards. Since allowed salbutamol preparations varied among different countries and among different sites within countries (i.e. metered dose inhaler versus powder inhaler), salbutamol use is expressed as $\mu \mathrm{g}$ salbutamol.day ${ }^{-1}$. Mean 
Table 3. - Effects of mometasone furoate administered by dry powder inhaler and budesonide Turbuhaler $(\mathbb{R})$ on pulmonary function, asthma symptoms and physician-evaluated response to therapy

\begin{tabular}{|c|c|c|c|c|}
\hline & \multicolumn{3}{|c|}{ MF DPI $\mu \mathrm{g}$ b.i.d } & \multirow{2}{*}{$\begin{array}{c}\text { BUD Turbuhaler@ } \\
\mu \mathrm{g} \text { b.i.d } \\
400\end{array}$} \\
\hline & 100 & 200 & 400 & \\
\hline Subjects $n$ & 185 & 176 & 188 & 181 \\
\hline \multicolumn{5}{|l|}{ Pulmonary Function } \\
\hline FEV1 L & $0.10 \pm 0.03$ & $0.16 \pm 0.03 *$ & $0.16 \pm 0.03 *$ & $0.06 \pm 0.03$ \\
\hline Endpoint FEV1 \% pred & $79.6 \pm 1.1$ & $81.6 \pm 1.2 *$ & $83.0 \pm 1.2^{+}, *$ & $77.9 \pm 1.1$ \\
\hline FVC L & $0.07 \pm 0.04$ & $0.16 \pm 0.04$ & $0.15 \pm 0.04$ & $0.06 \pm 0.04$ \\
\hline PEFa.m. L. $\min ^{-1}$ & $18.20 \pm 5.3$ & $37.84 \pm 5.4+$ & $37.31 \pm 5.2+$ & $24.75 \pm 5.3$ \\
\hline \multicolumn{5}{|l|}{ Patient Self Report-mean score } \\
\hline Wheezing a.m. & -0.07 & -0.17 & $-0.27^{+, *}$ & -0.10 \\
\hline Difficulty breathing a.m. & -0.10 & -0.20 & $-0.24^{+}$ & -0.14 \\
\hline Cough a.m. & -0.10 & -0.16 & -0.19 & -0.19 \\
\hline Nocturnal awakenings & -0.06 & -0.09 & -0.16 & -0.07 \\
\hline Salbutamol use in $\mu \mathrm{g} \cdot \mathrm{day}^{-1}$ & -45.86 & $-90.66^{*}$ & -72.13 & -33.90 \\
\hline Physician evaluated response to therapy & 2.43 & $2.33^{*}$ & $2.25^{*}$ & 2.53 \\
\hline
\end{tabular}

Values are presented as change from baseline to endpoint (last treatment visit) \pm SEM, with the exception of the endpoint FEV $1 \%$ pred. FEV1: forced expiratory volume in one second; ${ }^{+}: \%$ pred: per cent predicted; FVC: forced vital capacity; PEFa.m.: peak expiratory flow in the morning; MF DPI: mometasone furoate administered by dry powder inhaler; BUD: budesonide. *: $\mathrm{p}<0.05$ compared with BUD Turbuhaler $\AA^{\circ} 400 \mu \mathrm{g}$ b.i.d.; +: $\mathrm{p}<0.05$ compared with MF DPI $100 \mu \mathrm{g}$ b.i.d.

salbutamol use at baseline was very similar among the treatment groups (table 2). Based upon the reduction in mean total daily salbutamol use at the endpoint, patients treated with MF DPI $200 \mu \mathrm{g}$ b.i.d required significantly less salbutamol than did patients treated with BUD Turbuhalerß $400 \mu \mathrm{g}$ b.i.d (table 3).

Nocturnal awakenings requiring the use of salbutamol rescue medication were very low, and comparable, among patients in all treatment groups at baseline (table 2). Mean decreases in the number of nocturnal awakenings were observed for all treatment groups by the endpoint, with no significant differences between groups (table 3 ).

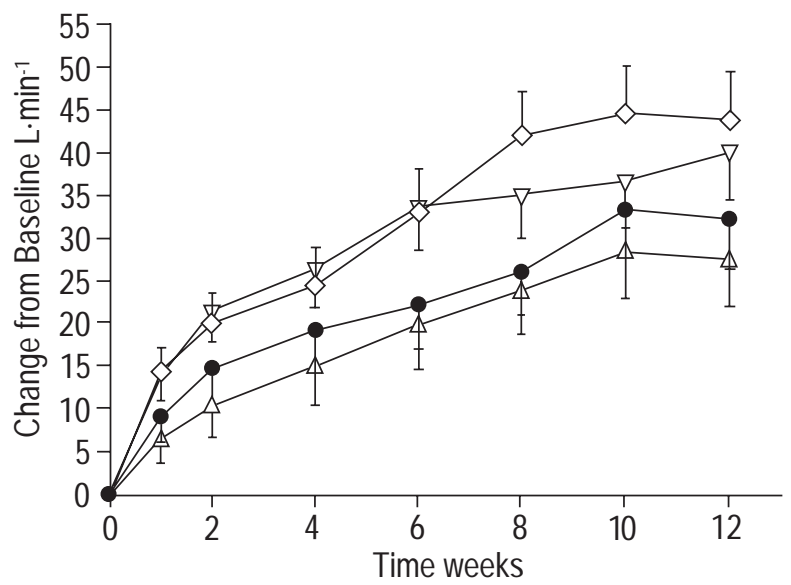

Fig. 2. - The effects of mometasone furoate (MF) b.i.d and budesonide (BUD) Turbuhaler $($ b.i.d on the mean change in morning peak expiratory flow rate (PEFa.m.). The changes for MF DPI $100 \mu \mathrm{g}, 200 \mu \mathrm{g}$, $400 \mu \mathrm{g}$ b.i.d and BUD Turbuhaler® $400 \mu \mathrm{g}$ b.i.d are indicated at Weeks 1, 2, 4, 6, 8, 10 and 12. $(\triangle)$ : MF $100 \mu \mathrm{g}$ b.i.d; $(\nabla)$ : MF $200 \mu \mathrm{g}$ b.i.d; $(\diamond)$ : MF $400 \mu \mathrm{g}$ b.i.d; (O): BUD $400 \mu \mathrm{g}$.Values are represented as mean \pm SEM.

\section{Physician evaluation of response to therapy}

The physicians evaluated the response to therapy for every patient at each treatment visit, by comparing symptom levels to those at baseline. At the endpoint, the percentage of patients considered to be much improved or improved was comparable in the MF DPI $100 \mathrm{mg}$ b.i.d (60\%), MF DPI $200 \mu \mathrm{g}$ b.i.d (63\%), and MF DPI $400 \mu \mathrm{g}$ b.i.d $(65 \%)$ treatment groups, and lower in the BUD Turbuhalerß $400 \mu \mathrm{g}$ b.i.d (50\%) group. The differences, expressed as mean scores, are significant for both the MF DPI $400 \mu \mathrm{g}$ b.i.d and MF DPI $200 \mu \mathrm{g}$ b.i.d treatment groups compared with the BUD Turbuhalerß $400 \mu \mathrm{g}$ b.i.d group (table 3 ).

\section{Evaluation of safety}

All treatments were well tolerated, and no unusual or unexpected adverse events were reported. Most events were mild to moderate in severity and none were life threatening. The most common adverse events, reported by $\geq 10 \%$ of patients in any treatment group, included headache, pharyngitis, viral infection, and rhinitis.

The incidence of adverse events judged by investigators to be related to treatment was similar for all treatment groups $(17-20 \%)$. The most common treatment-related adverse events were headache (4-8\%), pharyngitis (4 $5 \%$ ), and dysphonia (2-5\%). Dysphonia was distributed among the groups as $\mathrm{n}=8,5,9$, and 4 in the MF DPI 100, 200, 400, $\mu \mathrm{g}$ b.i.d and BUD Turbuhaler $\mathbb{R}$ ) $400 \mu \mathrm{g}$ b.i.d groups, respectively. Oral candidiasis was uncommon in this study, reported by only 16 patients overall, and had a similar incidence among the treatment groups $(n=4,6,4$, and 3 in the MF DPI 100, 200, 400, $\mu \mathrm{g}$ b.i.d and BUD Turbuhalerß $400 \mu \mathrm{g}$ b.i.d groups, respectively). Oral candidiasis was predominantly mild to moderate in severity.

Serious adverse events were noted for 11 patients (one in the screening period, two in the MF DPI $100 \mu \mathrm{g}$ b.i.d 
treatment group, three in the MF DPI $200 \mu \mathrm{g}$ b.i.d group, two in the MF $400 \mu \mathrm{g}$ b.i.d group, and three in the BUD Turbuhalerß $400 \mu \mathrm{g}$ b.i.d group). None of these events was considered to be related to the treatment.

Overall, 17 patients $(2 \%)$ did not complete the treatment because of adverse events. These included six $(3 \%)$ in the MF DPI $100 \mu \mathrm{g}$ b.i.d group, one $(<1 \%)$ in the MF DPI 200 $\mu \mathrm{g}$ b.i.d group, three (2\%) in the MF DPI $400 \mu \mathrm{g}$ b.i.d

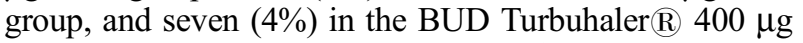
b.i.d group. Treatment was interrupted in some patients because of an adverse event $(n=16)$. In the majority of cases, the events causing interruption were thought to be unrelated to treatment. No clinically relevant changes in vital signs or physical exams were observed from baseline to endpoint for any treatment group. The few clinically significant changes in laboratory values were thought to be unrelated to study medication. No ECG abnormalities were reported that indicated any clinically significant effect of investigational treatment.

Compared with screening values, when patients were maintained on their previous inhaled corticosteroid, all four treatment groups demonstrated a slight increase in 08:00 h plasma cortisol levels at week 12 . At screening, median plasma cortisol levels were $13.3 \mu \mathrm{g} \cdot \mathrm{dL}^{-1}, 13.4 \mu \mathrm{g} \cdot \mathrm{dL}^{-1}$, $12.8 \mu \mathrm{g} \cdot \mathrm{dL}^{-1}$, and $13.6 \mu \mathrm{g} \cdot \mathrm{dL}^{-1}$ for the MF DPI 100, 200, and $400 \mu \mathrm{g}$ b.i.d groups and the BUD $400 \mu \mathrm{g}$ b.i.d group, respectively. At week 12, median plasma cortisol levels (percent change) were $13.7 \mu \mathrm{g} \cdot \mathrm{dL}^{-1}(+3 \%), 13.7 \mu \mathrm{g} \cdot \mathrm{dL}^{-1}$ $(+5 \%), 12.9 \mu \mathrm{g} \cdot \mathrm{dL}^{-1}(+2 \%)$, and $14.2 \mu \mathrm{g} \cdot \mathrm{dL}^{-1}(+9 \%)$ for the MF DPI 100, 200, and $400 \mu$ g b.i.d groups and the BUD $400 \mu \mathrm{g}$ b.i.d group, respectively. There were no significant differences in cortisol values among treatment groups when compared at screening or week 12 .

\section{Discussion}

In the first such comparison of its kind, doses of MF 200 $\mu \mathrm{g}$ b.i.d and $400 \mu \mathrm{g}$ b.i.d administered by a novel breathactuated DPI were both significantly more effective than a $400 \mu \mathrm{g}$ b.i.d dose of BUD Turbuhaler $\mathbb{R}$ in patients with moderate persistent asthma who required daily use of inhaled corticosteroids to control their asthma. The $400 \mu \mathrm{g}$ b.i.d dose of BUD administered by Turbuhaler $(\mathbb{R}$ was comparable in effectiveness to a $100 \mu \mathrm{g}$ b.i.d dose of MF DPI for most of the parameters examined. This 12 -week multicentre study extends the finding of previous studies $[12,13]$ which demonstrated MF DPI to be effective and well tolerated. In the present study, MF DPI was directly compared to a clinically effective and widely used inhaled glucocorticoid, BUD Turbuhaler $\mathbb{R}$. Treatment with MF DPI, at both the $200 \mu \mathrm{g}$ and $400 \mu \mathrm{g}$ b.i.d doses, significantly improved measures of pulmonary function, and to a greater extent than treatment with BUD Turbuhaler $\mathbb{R}$ $(400 \mu \mathrm{g}$ b.i.d), within the first two weeks of the study. Since all patients in the study were using recommended (with label) doses of prescribed inhaled corticosteroids to control their asthma prior to beginning this trial, the additional improvement obtained with MF DPI treatment over the previous therapeutic regimen is considered to be clinically meaningful. Some of this improvement may have resulted from the increased compliance that occurs when patients enter a clinical study.
BUD Turbuhaler $\mathbb{R}$, the active control comparator used in this study, has been extensively investigated and has been shown to be effective in controlling asthma in adults [20-22]. In the current study, BUD was delivered via a Turbuhaler ( $\mathrm{R}$ at a dose of $400 \mu \mathrm{g}$ b.i.d, which is a commonly prescribed dose for patients with moderate persistent asthma. The effectiveness of this dose was assessed against the identical dose of MF DPI, as well as two lower doses of MF DPI. At half of the total daily dose of BUD Turbuhaler $\AA$, MF DPI $200 \mu \mathrm{g}$ b.i.d provided significant improvement compared with BUD in several asthma-related measures, including changes in FEV1, \% pred FEV1, the amount of rescue medication (salbutamol) required, and physicians' evaluation of response to therapy. Based on the outcomes measured in this study, the results obtained with BUD Turbuhaler $\mathbb{R}$ (400 $\mu \mathrm{g}$ b.i.d) were most comparable to MF DPI $100 \mu \mathrm{g}$ b.i.d. These findings suggest that MF DPI is more potent than BUD Turbuhaler $\mathbb{R}$, which is in agreement with in vitro findings $[4,5,7]$. However, only one dose of BUD was used in this study, and it is possible that higher doses of BUD would show an efficacy comparable to that of MF.

Clinical responses to inhaled corticosteroids generally fail to show significant differences over dose ranges of $\geq$ four-fold [14]. In the present study, some endpoint measures did detect differences with dosages of MF that were only two-fold to four-fold higher than the lowest dose.

A significantly greater improvement in the a.m. PEF scores were detected for treatment with MF DPI $200 \mu \mathrm{g}$ and $400 \mu \mathrm{g}$ b.i.d compared with the MF DPI $100 \mu \mathrm{g}$ b.i.d. The improvement in the mean p.m. PEF score of the MF DPI $400 \mu \mathrm{g}$ b.i.d group was also significantly different from that of the MF DPI $100 \mu \mathrm{g}$ b.i.d group. Significant differences were noted in several other measures when the MF DPI $100 \mu \mathrm{g}$ b.i.d group was compared with the MF DPI $400 \mu \mathrm{g}$ b.i.d group. This suggests that, for some measurements, the study was able to detect differences between the lowest and highest MF DPI dosages. Furthermore, it appears that for the patients in this study with moderate persistent asthma, MF DPI $100 \mu \mathrm{g}$ b.i.d may represent the lower end of the therapeutically beneficial dose range.

In this study the MF 200 and $400 \mu \mathrm{g}$ b.i.d doses appeared comparable: MF DPI $200 \mu \mathrm{g}$ b.i.d provided a benefit equal to MF DPI $400 \mu \mathrm{g}$ b.i.d in the primary efficacy variable, FEV1, as well as for most other measures of pulmonary function assessed. This finding is consistent with the results of a previous dose-ranging study of MF [12]. There are reports that higher doses of an inhaled glucocorticoid may lead to a significant improvement in patients with moderate or severe asthma or those with stable disease $[22,23]$. In the present study, however, doubling the dose of MF DPI from 200 to $400 \mu \mathrm{g}$ b.i.d provided no additional benefit, and suggests that the upper threshold of therapeutic benefit may have been attained for this population of patients.

All doses of MF DPI were well tolerated in this study. Oral candidiasis was uncommon in this study and demonstrated a similar incidence among treatment groups. Most adverse events were mild to moderate in severity and were unrelated to the study drug. There were no differences among treatment groups in the overall incidence of adverse events. 
The very low bioavailability of MF taken by DPI, and low potential for hypothalamic-pituitary-adrenal (HPA) axis suppression have recently been shown using sensitive measures, such as 24-h area under the curve cortisol concentrations and adrenal response to stimulation [24]. A study using the same dosing range of MF as in the present report found plasma levels of MP to be less than 50 $\mathrm{pg} \cdot \mathrm{dL}^{-1}$ and found no evidence of suppression of cortisol response to cosyntropin stimulation [12]. It was not feasible to conduct similar HPA function measurements in this large international study. However, using the change in 08:00 h plasma cortisol concentrations from screening to week 12 , there were no clinically relevant differences among treatment groups. In addition, there was no evidence of HPA axis suppression in any group compared with the screening value, obtained while patients were maintained on their previous inhaled corticosteroid. Due to the lower sensitivity of the 08:00 $\mathrm{h}$ cortisol measurements, these results should be interpreted with caution. However, the results of this study suggest that, in this patient population, there is little potential for HPA axis suppression by MF or BUD at the doses tested.

To conclude, this 12-week study showed that inhaled mometasone furate, administered by a novel breathactuated DPI, was effective and well tolerated by adults and adolescents who were previously maintained on daily inhaled corticosteroids for treatment of moderate persistent asthma. Lower doses of mometasone furate administered by dry powder inhaler appears to be a more effective treatment for asthma than a clinically recommended daily dose of BUD Turbuhaler $\AA$. while providing a comparable degree of safety.

Acknowledgements. The authors would like to thank the following individuals who participated in this study: C.G. DiBartolo; G. Ramon; J. Wilson and E.H. Walters; K. Chapman; W. Yang; P. Larivee; J-L. Malo; P. Small; M.T. Newhouse; C.B. Malig; H. Giraldo; E.G. Cadena; V. Kinnula; A. Granados; N. Rojas; S.J. Mol; J. Westbroek; U. Aasebo; F. Wammer; B. de Almeida; M.G. Vaz; J. Hedner; L. Rosenhall; J.T. Calvete; J. LopezAntonini; L. Lindemann; H. Bisping-Arnold; P. Patel; A.V. Pais; N. Feldman; J. Guerra; S. Castillo; V. M. Almeida; J. Roa; J-P. Ouellet; P. Bihet; A. Sabbah; P. Coulet; P. Couturier; J-C. Farouz; M. Grosclaude; F. Marmouz; C. Pison; F. Wessel; P. Kardos; B. Nothofer; E. Scheer; H. Sudhoff; L.V. Versen; F. Warlies.

\section{References}

1. National Heart Lung and Blood Institute. National Asthma Education and Prevention Program, Expert Panel Report 2. Guidelines for the diagnosis and management of asthma. Bethesda, MD: US Department of Health and Human Services; 1997. Report No.: 974051.

2. Global Initiative for Asthma. Global strategy for asthma management and prevention NHLBI/WHO workshop report. Bethesda, MD: National Institutes of Health, National Heart, Lung and Blood Institute; 1995. Report No.: 95-3659.
3. The British Thoracic Society, The National Asthma Campaign, Royal College of Physicians of London in association with the General Practitioner in Asthma Group, et al. The British guidelines on asthma management 1995 review and position statement. Thorax 1997; 52: S1S21.

4. Umland SP, Nahrebne DK, Razac S, et al. The inhibitory effects of topically active glucocorticoids on IL-4, IL-5, and interferon-gamma production by cultured primary CD4+ T cells. J Allergy Clin Immunol 1997; 100: 511519.

5. Crocker IC, Church MK, Newton S, Townley RG. Glucocorticoids inhibit proliferation and interleukin-4 and interleukin- 5 secretion by aeroallergen-specific Thelper type 2 cell lines. Ann Allergy Asthma Immunol 1998; 80: 509-516.

6. Barton BE, Jakway JP, Smith SR, Siegel MI. Cytokine inhibition by a novel steroid, mometasone furoate. Immunopharmacol Immunotoxicol 1991; 13: 251-261.

7. Smith CL, Kreutner W. In vitro glucocorticoid receptor binding and transcriptional activation by topically active glucocorticoids. Arzneimittelforschung 1998; 48: 956960 .

8. Drouin $\mathrm{M}$, Yang $\mathrm{WH}$, Bertrand $\mathrm{B}$, et al. Once daily mometasone furoate aqueous nasal spray is as effective as twice daily beclomethasone dipropionate for treating perennial allergic rhinitis patients. Ann Allergy Asthma Immunol 1996; 77: 153-160.

9. Graft D, Aaronson D, Chervinsky P, et al. A placebo- and active-controlled randomized trial of prophylactic treatment of seasonal allergic rhinitis with mometasone furoate aqueous nasal spray. J Allergy Clin Immunol 1996; 98: 724-731.

10. Hebert JR, Nolop K, Lutsky BN. Once-daily mometasone

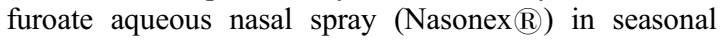
allergic rhinitis: an active- and placebo-controlled study. Allergy 1996; 51: 569-576.

11. Kenyon D, Yang TT, Kaufman A, et al. Comparison of mometasone furoate dry powder inhaler (MF-DPI) with Turbohaler using a new methodology for in vitro analysis of inhaler performance. Eur Respir J 1999; 14: $291 \mathrm{~S}$.

12. Bernstein DI, Berkowitz RB, Chervinsky P, et al. Doseranging study of a new steroid for asthma: mometasone furoate dry powder inhaler. Respir Med 1999; 93: 603-61.

13. Nayak AS, Banov C, Corren J, et al. Once-daily mometasone furoate dry powder inhaler in the treatment of patients with persistent asthma. Ann Allergy Asthma Immunol 2000; 84: 417-424.

14. Pedersen S, O'Byrne P. A comparison of the efficacy and safety of inhaled corticosteroids in asthma. Allergy 1997; 52: 1-34.

15. Hanania NA, Chapman KR, Kesten S. Adverse effects of inhaled corticosteroids. Am J Med 1995; 98: 196-20.

16. Kamada AK, Szefler SJ, Martin RJ, et al. Issues in the use of inhaled glucocorticoids. The Asthma Clinical Research Network. Am J Respir Crit Care Med 1996; 153: 17391748.

17. Price JF. Inhaled corticosteroids: clinical relevance of safety measures. Pediatr Pulmonol Suppl 1997; 15:40-45.

18. Crapo RO, Morris AH, Gardner RM. Reference spirometric values using techniques and equipment that meet ATS recommendations. Am Rev Respir Dis 1981; 123: 659-664.

19. Polgor G, Promadhat V. Pulmonary function testing in children: techniques and standards. Philadelphia, WB Saunders, 1971.

20. Aaronson D, Kaiser H, Dockhorn R, et al. Effects of 
budesonide by means of the Turbuhaler on the hypothalamic-pituitary-adrenal axis in asthmatic subjects: a dose-response study. J Allergy Clin Immunol 1998; 101: 312-319.

21. Nelson HS, Bernstein IL, Fink J, et al. Oral glucocorticosteroid-sparing effect of budesonide administered by Turbuhaler: a double-blind, placebo-controlled study in adults with moderate-to-severe chronic asthma. Pulmicort Turbuhaler Study Group. Chest 1998; 113: 264-1271.

22. Busse WW, Chervinsky P, Condemi J, et al. Budesonide delivered by Turbuhaler is effective in a dose-dependent fashion when used in the treatment of adult patients with chronic asthma. J Allergy Clin Immunol 1998; 101: 457463.

23. Carpentiere G, Marino S, Castello F, Baldonza C, Bonanno CT. Dose-related effect of beclomethasone dipropionate on airway responsiveness in asthma. Respiration 1990; 57: 100-103.

24. Affrime MB, Kosoglou T, Thonoor CM, Flannery BE, Herron J. Mometasone furoate has minimal effects on the hypothalamic-pituitary-adrenal axis when delivered at high doses. Chest 2000; (in press). 